\title{
COMMISSION 36: THEORY OF STELLAR ATMOSPHERES (THÉORIE DES ATMOSPHÈRES STELLAIRES)
}

\author{
Report of Meetings, 19, 20, 25 and 26 August 1970
}

President: A. B. Underhill.

SECRETARY: A. L. T. Powell.

\section{Business Meeting}

The members learned with regret of the deaths of L. G. Henyey, J. Q. Stewart and M. H. Wrubel since the last General Assembly. The following additions were made to the Report of the Commission: A-1: - A set of models for F stars has been published by T. Kipper. A-8: - The nature of P Cygni stars has been discussed by L. Luud. C-4: - A more exact equation of radiative transfer has been derived from the basic equations of quantum electrodynamics by A. Sapar who has discussed the implications in detail. Computational techniques for plane-parallel radiative transfer based on mathematical semigroup properties of the operators for diffuse reflection and transmission in scattering atmospheres have been developed by Grant and Hunt. C-7: - Problems of extended stellar atmospheres and of stationary mass loss are being studied by Pustylnik and by Sapar and Viik. In addition analytical expressions for the redistributed intensity as a result of multiple Compton scattering in a spherical atmosphere is under study by Viik. As with the work reported previously, reference must be made to standard abstracting journals to find where these studies are published.

The new Organizing Committee drafted a set of by-laws for the Commission. These are given at the end of this report.

Plans for colloquia to be held in the next three years were discussed. Choice will be made by the new Organizing Committee from among the topics: The transfer of radiation and the formation of spectral lines in the presence of a magnetic field; Theoretical considerations of spectral line profiles; Interdisciplinary aspects of stellar atmospheres. The venues of Boulder, Nice and Istanbul were mentioned and either late spring or early autumn were considered to be the best time for such intensive working meetings.

In response to a resolution submitted by the Executive Committee to Commission 36 for consideration, the following resolution was approved: Commission 36 considers the physics of unstable stars to be a most important subject in which it is deeply interested. However, because the physics of unstable stars is basically the same as that of stable stars, it does not recommend the creation within one of the IAU Commissions of a subcommission on the physics of unstable stars. Commission 36 suggests that colloquia or symposia be held every few years to summarize the problems and results in the field of unstable stars.

The following resolution drafted by R. N. Thomas and J.C. Pecker was also approved for submission to the IAU Executive Committee:

For some years Commission 36 has realized the vital importance of small working colloquia and symposia on topical problems in stellar atmospheres to delineate problems and continually to re-examine the basis of theoretical models and diagnostic spectroscopy. A major part of such colloquia is lost without the prompt preparation and distribution of a set of inexpensive proceedings so that persons unable to attend the colloquia may still share their stimulus. This is particularly true for students, young researchers and mature persons just shifting their attention to another field.

Therefore, Commission 36 proposes to implement the following policy and it recommends and invites other Commissions of the IAU to join it:

(a) to encourage the Organizing Committee and the Editor of the Proceedings of each symposium and colloquium to utilize the staff and secretarial facilities of their institution to prepare quickly camera copy of the major summary papers and discussions for photo-offset reproduction. Paper quality and artistic appearance are negligible considerations; 
(b) to encourage collaborative efforts between governmental and institutional publication offices to publish simultaneous English and Russian language editions at a price such that each student can afford to purchase his own working copy.

To ease the problem of literature monitoring, the meeting approved the proposal by R. N. Thomas that a preprint, summary and reprint of all papers relevant to stellar atmospheres should be sent to the Commission President. These would then be collated yearly and a bibliography would be sent to each member and to anybody making a request to receive such information.

\section{COMMISSION 36 BY-LAWS}

1. The Organizing Committee is elected by members by ballot, from a list of candidates proposed by the members. The number of candidates is substantially larger than the number to be elected.

2. The possible subjects for next colloquia, symposia or other types of gathering sponsored by Commission 36 are proposed by the members and selected by the Organizing Committee.

3. Questions calling for decisions or statements on behalf of Commission 36 are normally first brought to the attention of the President, secondly evaluated by the Organizing Committee and then submitted to the members of Commission 36 for their approval.

\section{Scientific Meeting}

The first topic to be discussed was 'The problem of spectrum formation without the hypothesis of LTE: physical principles and mathematical techniques'. $\mathrm{R}$. N. Thomas introduced the subject with a review of the physical principles. A summary of his remarks follows:

A step-by-step summary of the physical principles introduced is given. Spectrum formation results from the interaction between energy states of matter and of the radiation field as the radiation diffuses outward through the stellar atmosphere. Such interaction is described by the structure and variation through the atmosphere of the source-function. Section I summarizes the general structure of the source-function, $S_{v}$, in terms of energy states of matter, their interaction among themselves and with the radiation field, and the explicit use of population and conservation equations in making specific the structure and variation of $S_{\nu}$. It also summarizes the physical and mathematical simplification resulting from imposing the conditions characterizing the classical atmosphere. Section II summarizes the conditions under which astronomical LTE, or LTE-R, results even when the condition of LTE-R is not a priori imposed; viz, collision domination, or homogeneity of radiation field under additional conditions. Section III summarizes the principles upon which a construction of the currently-used form of $S_{v}$ rests; its $v$-dependence; the relation between $S_{v}, J_{v}$, and so-called source- and sink-terms in thick, effectively-thin, and thin atmospheres; and the idea of the New Spectroscopy. Section IV discusses the computation of the values of state-parameters, or equivalently source-sink terms, throughout the atmosphere, trying carefully to distinguish between population and conservation-equation effects. The use of the Temperature-Control-Brackets, TCB, is briefly outlined, in connection with obtaining a physical picture.

A review of the mathematical techniques was then given by $\mathbf{L}$. H. Auer. In summary he said the following:

The mathematical difficulty of the non-LTE problem lies in the fact that we must find simultaneously and self-consistently: (1) the radiation field at each frequency, (2) the electron velocity distribution, and (3) the population of each level. Because the radiation field is not locally controlled, solution must proceed simultaneously at all depths. Modern methods of treating transfer problems enable one to solve any case in which the equation of transfer is linear in the radiation field. There are two classes of such techniques: those based on the integral equation formulation and those based on the differential equation formulation. Both reduce the continuous equations to a set of linear equations which may be directly solved for the source function (or radiation field) at all depths. Since not only the transfer equation but also the equation of statistical equilibrium must be satisfied, iteration is always necessary. In the simplest approach, the transfer equation is linearized 
and solved one transition at a time. With the new radiation field, new populations are computed and the process iterated to convergence. Alternatively, the entire set of equations, both transfer and statistical equilibrium, may be completely linearized, and the resulting system solved. To the extent that the equations are really linear, this scheme simultaneously and consistently finds all quantities. In complex problems it is easier to apply and more strongly convergent than the former method; however, each iteration requires much more time. It is possible that a mixed scheme involving linearization of several but not all transitions is optimal from the standpoint of computing time.

Four contributed papers were presented. A. G. Hearn considered the effects of gradients of the temperature and electron density on the departures from LTE for stellar absorption lines. The importance of considering the population of many levels in the atom was stressed.

The empirical determination of $S_{L}$ and $b_{L}$ as functions of $\tau$ for infrared solar lines was considered by $C$. de Jager and L. Neven for $C r$ lines in the Sun. The source function was found to follow the LTE value, but for some lines, the value of $b_{L}$ was much greater than unity.

Results of non-LTE atmosphere calculations given by L. H. Auer showed that the line profiles of HeII lines computed by non-LTE techniques developed by himself and Mihalas improved the agreement with observation. The discussion was closed by a contribution from A. Skumanich on the application of the Generalized Newton-Raphson method to non-LTE problems.

The second topic for this session was 'The Atmospheres of Cool Stars'. Y. Fujita discussed the theoretical problems of the atmospheres of cool stars and showed results of opacity calculations done at Tokyo for a wide range of molecules. Recent calculations of the opacity of $\mathrm{CN}$ in cool stars were presented by H. R. Johnson. These showed that this source of opacity was very significant when the $\mathrm{CN}$ molecule was dominant.

\section{Joint Scientific Meeting with Commission 29}

In this session, arranged by Commission 29, the following papers were read on the topic 'How to Determine Abudances':

A. B. Underhill: 'What Does One need to know to Determine Abundances'.

R. F. Griffin: 'How Accurate are Measurements of Equivalent Widths and Line Profiles'.

B. E. J. Pagel: 'Uses and Limitations of the Differential Curve-of-Growth Method'.

L. H. Aller: 'Use of Line Profiles in Abundance Work'.

R. Cayrel: 'Comparison of High Dispersion Studies with Scanner Work'.

A. Unsöld: 'General Survey of Recent Experimental and Theoretical Work on the Abundance Problem'.

The speakers reviewed the field of abundance determination outlining the errors involved and validity of the physical arguments used to interpret the observations as well as the effect of systematic errors in the observations. The accuracy obtained from curve-of-growth methods appears to match the deficiencies in model atmospheres, spectroscopic data and observations. Scanner measurements give results consistent with those from other methods but with some loss in accuracy. Movement towards agreement in the determination of the solar iron abundance from the different available methods has followed from a revision of the FeI and FeII $f$-values. The ramifications of this change on the physical properties of the solar atmosphere were outlined by Unsöld.

\section{Scientific Session}

The first half of this session was devoted to a discussion of solar abundances. L. H. Aller, the discussion leader, confined his introductory remarks mainly to the subject of spectrum synthesis. He outlined the methods, assumptions, limitations and results obtained by members of his group at the University of California. By judicious adjustment of certain free physical parameters in a model atmosphere, the computed spectrum can be made to fit the observations in most cases. This approach can be used to determine the abundance of low-abundance elements that only occur in blended lines. Discussion followed concerning the justification of adjusting the various parameters 
which in some cases gave unrealistic values; in other cases, it was impossible to reproduce all the observed features of the spectrum. J. C. Pecker then gave an account of his recent work on the polarization variation of the solar continuum with wavelength. The results, independent of $f$-values and damping constant, indicated that the metal composition of the Sun was at least five times the GMA values. The recent upward revision of the magnesium, silicon and iron abundances makes this result more plausible. G. Elste showed how the use of high excitation Fe II lines for the curveof-growth analysis of A stars has revealed a need for a revision of the $f$-values. These had given a large scatter on the curve and an anomalously low excitation temperature. A correction, dependent on excitation potential, was found by comparing the original $f$-values used in the analysis with those given by Garz and Kock. Application of this correction removed the scatter and temperature anomaly.

The second half of this session was devoted to contributions on 'The Problem of Spectrum Formation without the Hypothesis of LTE' that had been received since the announcement of the first session. R. C. Altrock outlined a method of 'Empirical Analysis of Line Profiles'. This was based on the solution for the source function from three lines of one multiplet. Preliminary results on the infrared oxygen triplet showed the source function to be in agreement with those of BCA. The theory of radiative transfer in a two component atmosphere was presented by E. H. Averett. This involved the calculation of the source function in the presence of material motion in hexagonal columns. P. R. Wilson described work undertaken by some of his colleagues on the vertical and horizontal fluctuations of temperature, density and opacity in the solar photosphere. Solution for the source function followed the Feautrier method. From a comparison of old and young Of stars, S. R. Heap described the discrepancy that had been found between the Zanstra temperatures and those derived from model atmospheres. This cannot be resolved by the use of classical models. In a discussion of the formation of the Lyman continuum, W. Kalkofen presented the results of his and R. W. Noyes' work on the construction of a model solar atmosphere that predicted the dissimilar colour and brightness temperatures observed. This model is based on the detailed balancing of three bound levels of hydrogen and the continuum. Recent work on the computation of a non-LTE line-blanketed model was given by R. G. Athay. Line blanketing is taken into account by using a representative atom with three levels and two line transitions. The model atmosphere was found to give good agreement with solar observations. 